\title{
Forensic examination of effects of rheumatoid arthritis on handwriting characteristics
}

\author{
Komal Saini* D, Bhavya Sharma and Manpreet Kaur
}

\begin{abstract}
Background: Rheumatoid arthritis is known to produce deformity of the joints and muscles which can affect the handwriting of an individual significantly. Examination of such handwriting specimens can become a challenging task for the document examiners. This can affect the accuracy of their opinions upon the related cases. Thus, the present study was conducted to determine the effects of rheumatoid arthritis on handwriting characteristics qualitatively and statistically. The study also included qualitative analysis and comparison of line quality defects of copied writings and writings affected by rheumatoid arthritis.
\end{abstract}

Results: Handwriting characteristics were analyzed qualitatively and statistically in old writings vs affected writings. All these handwriting characteristics were also analyzed qualitatively in copied writings vs affected writings. The results of the study showed significant deteriorating effects of rheumatoid arthritis on almost all handwriting characteristics, except for characteristics such as slant, retouching, and overwriting. The pictorial appearance of letters in the writings executed before and after the onset of illness was even observed to appear different altogether in case of some individuals with severely affected writings. Moreover, the analysis of copied writings indicated the execution of writing by a different person. Handwriting characteristics such as slant, spacing, and alignment were observed to show insignificance in analyzing differences between copied and genuine writings affected by the illness.

Conclusion: It has been concluded that rheumatoid arthritis causes deterioration of letters, tremor, disturbed rhythm, and a huge variation in other handwriting characteristics. The copied writings have also presented line quality defects like artificial tremor, hesitations, retouching, and unnatural pen lifts at all wrong places. The determination of these peculiarities may assist the document examiners in the differentiation of copied and affected writings.

Keywords: Questioned document examination, Document examiners, Handwriting characteristics, Rheumatoid arthritis, Copied writings

\section{Background}

Handwriting is an intricate activity that requires extensive neuromuscular coordination. Physiological illnesses like rheumatoid arthritis (RA) are known to drastically affect the muscular control involved in the process of handwriting (Huber and Headrick 1999). RA primarily

\footnotetext{
* Correspondence: komal2saini@yahoo.com

Department of Forensic Science, Punjabi University, Patiala, Punjab, India
}

affects small joints and most RA patients develop stiffness, pain, swelling, and rigidity in the hand, fingers, and wrist joints which are functional in writing. This leads to severe deterioration in the quality of handwriting, thus, rendering the authenticity of the process debatable (Saini 2015). Document examiners are required to acquire precise knowledge of various effects of such an illness on the handwriting characteristics of a patient and how they differ from forged writings.

\section{Springer Open}

(c) The Author(s). 2021 Open Access This article is licensed under a Creative Commons Attribution 4.0 International License, which permits use, sharing, adaptation, distribution and reproduction in any medium or format, as long as you give appropriate credit to the original author(s) and the source, provide a link to the Creative Commons licence, and indicate if changes were made. The images or other third party material in this article are included in the article's Creative Commons licence, unless indicated otherwise in a credit line to the material. If material is not included in the article's Creative Commons licence and your intended use is not permitted by statutory regulation or exceeds the permitted use, you will need to obtain permission directly from the copyright holder. To view a copy of this licence, visit http://creativecommons.org/licenses/by/4.0/. 
While multiple studies have elucidated the immense effect of old age and illness on handwriting (Osborn 1929; Quirke 1930; Brewester 1932; Baker 1950; Hilton 1956, 1977; Harrison 1966; Miller, 1987; Saini 2015), the present study aims to analyze the effects of rheumatoid arthritis on handwriting characteristics, both qualitatively and statistically, and determine whether it is possible to compare the concerned writings executed under the influence of rheumatoid arthritis with those written before the onset of the illness. The study also includes qualitative analysis and comparison of the copied writings with the handwriting samples of people affected by RA to determine the characteristics which can be used in the identification and differentiation of these writings.

\section{Methods}

\section{Collection of handwriting samples}

Sixty patients diagnosed with RA were included in the study; the patients were contacted by visiting various orthopedic hospitals, physiotherapy clinics, and related institutions. After a comprehensive explanation of the study, handwriting samples were collected from the interested patients who provided written informed consent. Demographic details of the patients were recorded on a basic information sheet (Table 1).

As per the study objectives, two sets of handwriting samples - genuine writings and copied writings - were collected and prepared. Genuine writings were collected from RA patients that included handwriting samples executed in 2 different health conditions - before and after the onset of RA. All RA patients were requested to provide samples of handwriting executed before the onset of RA $(\mathrm{OH})$, that is, old writings from their previous records. Sixty old writings were collected that served as standard samples or control specimens. All the subjects were then instructed to write the same content (as written in their old writings) for standardization of the text. The old writings were placed in front of the subjects to help them write the same content and no instructions were given regarding the style or manner of writing. Thus, another set of writings, that is, sixty affected writings $(\mathrm{AH})$, were collected from the patients to be compared with their old writings.

Copied writings $(\mathrm{CH})$ of all the affected writings were prepared with the help of a skilled volunteer to evaluate and identify different features of defective handwriting between the copied and affected writings. Thus, an equal number of copied writings $(n=60)$ were prepared for the present study. No instructions were given to the volunteer regarding the manner of copying.

Thus, a total of 120 genuine writings and 60 copied writings were obtained and analyzed.

\section{Analysis}

All the handwriting samples, namely $\mathrm{OH}, \mathrm{AH}$, and $\mathrm{CH}$ were compared as $\mathrm{OH}$ vs $\mathrm{AH}$ and $\mathrm{CH}$ vs $\mathrm{AH}$; they were analyzed and differentiated qualitatively on the basis of change in quality, presence/absence, and increased/decreased number of handwriting characteristics, such as line quality features (rhythm, tremor, pen lifts, retouching or overwriting), letter formation, writing speed, omission of letters or parts, simplification or abbreviation of letters or parts, initial and terminal strokes, connecting strokes, overall size, slant, spacing, and alignment.

Statistical analysis was conducted using IBM $^{\circ}$ SPSS $^{\circ}$ Statistics software. For statistical analysis, the handwriting characteristics were coded along with variables $(\mathrm{OH}$

Table 1 Demographic details of rheumatoid arthritis subjects

\begin{tabular}{llll}
\hline Parameters & Groups & Number of subjects & \%Age \\
\hline Age & Below 40 & 5 & 12 \\
& $41-50$ & 15 & 20 \\
& $51-60$ & 15 & 25 \\
& $61-70$ & 11 & 18.3 \\
& $71-80$ & 10 & 16.6 \\
Gender & $81-90$ & 7 & 11.6 \\
Educational qualification & Male & 18 & 30 \\
& Female & 42 & 70 \\
Occupation & Matric or below matric & 20 & 33.33 \\
& Higher secondary education & 15 & 25 \\
& Graduation or above & 25 & 41 \\
& Self-employed & 12 & 43.33 \\
\hline
\end{tabular}

Details of all RA subjects participating in the study, showing different parameters, groups, and number of subjects belonging to each group 
and $\mathrm{AH}$ ) and were then divided into 2 categories, "0" and "1", according to the quality of characteristics observed (Table 2) (Saini and Kaur 2019). The handwriting samples showing characteristics with good line quality and constancy were placed into the "0" category, whereas characteristics with poor or disturbed line quality and variation were placed into the " 1 " category.

The two samples of genuine writings, $\mathrm{OH}$ and $\mathrm{AH}$, were compared using a paired $t$-test. The null hypothesis $\left(H_{0}\right)$ stated that there will be no difference in handwriting features of "OH" and "AH" samples $\left(H_{0}=\mu_{1}=\mu_{2}\right)$. The alternative hypothesis $\left(H_{1}\right)$ was that the true mean difference between the two paired samples would not be zero $\left(H_{1}=\mu_{1} \neq \mu_{2}\right)$ (the population mean for $\mathrm{OH}$ is represented as " $\mu_{1}$ " and for $\mathrm{AH}$ is represented by " $\mu_{2}$ "). A $p$ value of $<0.05$ was chosen to determine statistical significance; if $p<0.05, H_{0}$ is rejected, implying that RA will have significant deteriorating effects on the handwriting characteristics.

\section{Results}

The results of the qualitative and statistical analysis have been outlined and discussed in the following section.

\section{Qualitative analysis}

The following observations were made during the qualitative analysis and comparison of both $\mathrm{OH}$ vs $\mathrm{AH}$ and $\mathrm{CH}$ vs $\mathrm{AH}$ :

\section{Line quality features \\ Rhythm}

The overall deterioration of rhythm was observed in 53 out of $60 \mathrm{AH}$ samples. The letter strokes in these writings indicated a lack of rhythm - angularity and a change in the direction of strokes (Fig. 1a) was evident in the $\mathrm{AH}$ samples.

The $\mathrm{CH}$ samples also showed an overall deterioration of rhythm; however, these were due to the occurrence of hesitations at all wrong places in the strokes (Fig. 1b). This was also indicated by the copiers' difficulty in forming certain unknown forms and combinations in some parts of the writing, while the other parts of writing showed strokes formed with freedom and smoothness.

\section{Tremor}

Tremor was observed in 49 of the $60 \mathrm{AH}$ samples as compared to their corresponding $\mathrm{OH}$ samples. Tremor was observed in the form of involuntary wavy strokes at almost every type of stroke (including numerals) due to a change in the writing impulse of the affected hand and the resultant AH (Fig. 2a).

On the other hand, $\mathrm{CH}$ samples showed carefully drawn tremulous strokes in the form of hesitations and constrained strokes of a high degree that were discovered at all wrong and unnatural places of the letters (Fig. $2 b$ ). Thus, the tremor observed in copied writings was representative of artificial tremor.

Table 2 Categorization and coding of handwriting characteristics along selected variables

\begin{tabular}{|c|c|c|c|c|c|}
\hline \multirow{2}{*}{$\begin{array}{l}\text { S. } \\
\text { No. }\end{array}$} & \multirow{2}{*}{$\begin{array}{l}\text { Handwriting } \\
\text { characteristics }\end{array}$} & \multirow[t]{2}{*}{ Code } & \multirow{2}{*}{$\begin{array}{l}\text { Codes along } \\
\text { variables }\end{array}$} & \multicolumn{2}{|l|}{ Categorization of characteristics } \\
\hline & & & & Category $1(0)$ & Category 2 (1) \\
\hline 1. & Rhythm & RM & RM_OH/RM_AH & $0=$ Smooth & $1=$ Deteriorated \\
\hline 2. & Tremors & TM & TM_OH/TM_AH & $0=$ Absent & $1=$ Present \\
\hline 3. & Pen lifts & PN & PN_OH/PN_AH & $0=$ Absent & $1=$ Present \\
\hline 4. & Retouching/overwriting & $\mathrm{RO}$ & $\mathrm{RO} \_\mathrm{OH} / \mathrm{RO} \_\mathrm{AH}$ & $0=$ Absent & $1=$ Present \\
\hline 5. & Letter formation & $\mathrm{DL}$ & $\mathrm{DL} \_\mathrm{OH} / \mathrm{DL} \_\mathrm{AH}$ & $0=$ Absent & $1=$ Present \\
\hline 6. & Overall writing speed & WS & WS_OH/WS_AH & $0=$ Fast & $1=$ Slow \\
\hline 7. & Omission of letters/parts & $\mathrm{OM}$ & OM_OH/OM_AH & $0=$ Absent & $1=$ Present \\
\hline 8. & Simplification/abbreviation & SM & SM_OH/SM_AH & $0=$ Absent & $1=$ Present \\
\hline 9. & Initial and terminal strokes & IT & IT_OH/IT_AH & $0=$ Tapered & $1=$ Tremulous/blunt \\
\hline 10. & Connecting strokes & NC & $\mathrm{NC} \_\mathrm{OH} / \mathrm{NC} \_\mathrm{AH}$ & $0=$ Smooth/curved & $1=$ Tremulous/angular \\
\hline 11. & Overall size & $\mathrm{SI}$ & SI_OH/SI_AH & $0=$ Constant & $1=$ Increase $/$ decrease \\
\hline 12. & Slant & $\mathrm{SL}$ & SL_OH/SL_AH & $0=$ Vertical/forward/backward/mixed & $1=$ Variation \\
\hline 13. & Spacing & SP & SP_OH/SP_AH & $0=$ Narrow $/$ wide $/$ constant & $1=$ Variation \\
\hline 14. & Alignment & AG & AG_OH/AG_AH & $0=$ Horizontal/uphill/downhill/mixed & $1=$ Variation \\
\hline \multicolumn{6}{|c|}{ Variables } \\
\hline 1. & Pre-condition (before RA) & $\mathrm{OH}$ & & & \\
\hline 2. & Post-condition (after RA) & $\mathrm{AH}$ & & & \\
\hline
\end{tabular}


Sain et al. Egyptian Journal of Forensic Sciences

(2021) 11:19

Page 4 of 10

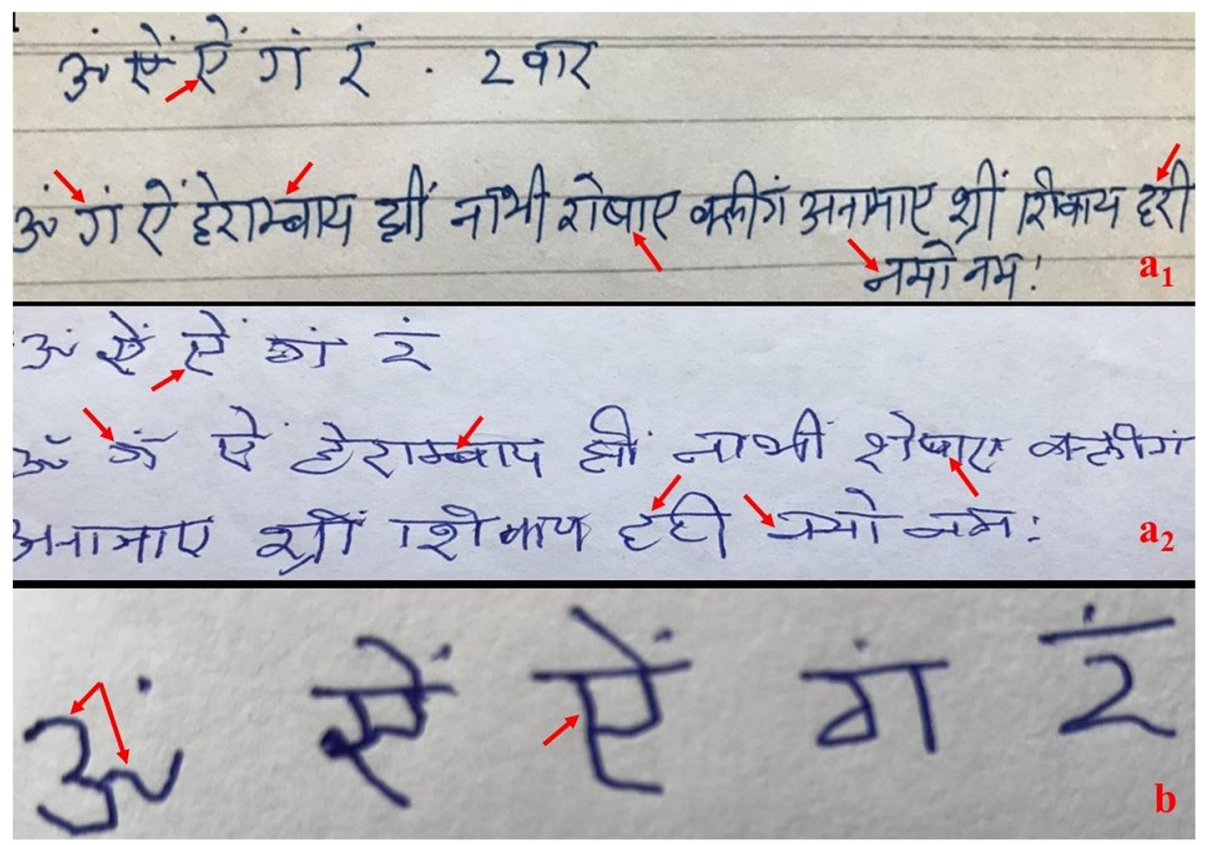

Fig. 1 Rhythm. $\mathbf{a}_{\mathbf{1}}$ Handwriting before onset of RA showing rhythmic strokes of writing. $\mathbf{a}_{\mathbf{2}}$ Non-rhythmic writing strokes in RA patient. $\mathbf{b}$ Hesitating strokes at wrong places in copied writing

Pen lifts

Pen lifts were observed in only 29 out of the $60 \mathrm{AH}$ samples. There was inconsistency in the pen lifts in the majority of affected writings as evident from both the occurrence at some places and lack of pen lifts in the same writing. The samples written in the Devanagari script demonstrated significantly high pen lifts during the formation of "Maras" and loops (Fig. Ba). Some writings showed bold rejoining of strokes at places of pen lifts, whereas some of the $\mathrm{AH}$ writings even reflected no extra efforts to rejoin the leftover strokes.
Additionally, the difficulty in continuing the writing was found to cause pen lifts in the AH samples and was indicative of muscular stiffness and a lack of movement control.

Extensive pen lifts were seen at almost all unnatural places in a considerable number of $\mathrm{CH}$ samples as compared to the affected writings. This was also evident from the copiers' conscious efforts to copy the letters of genuine writings. Also, copied writings showed peculiar pen lifts for joining the leftover strokes by drawing the

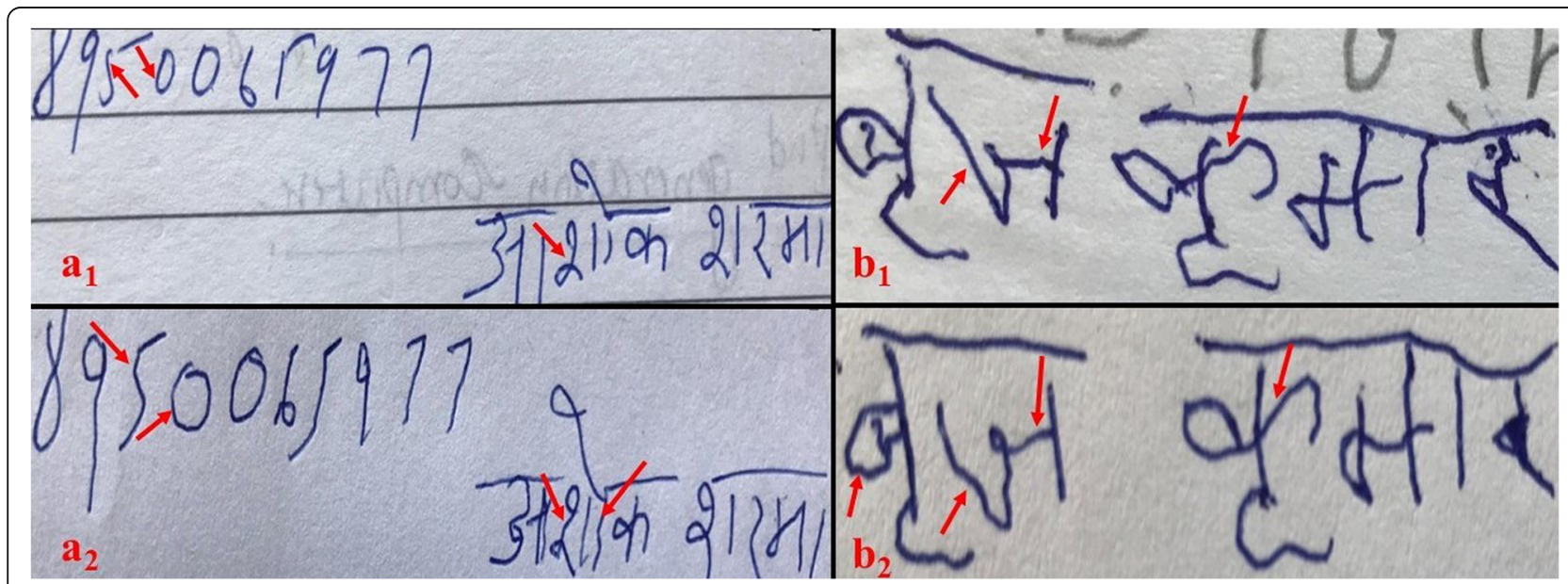

Fig. $\mathbf{2}$ Tremor. $\mathbf{a}_{\mathbf{1}}$ Absence of tremor in writing before onset of RA. $\mathbf{a}_{\mathbf{2}}$ Tremor in handwriting of RA patient. $\mathbf{b}_{\mathbf{2}}$ Artificial tremor observed at unnatural places in copied writing 


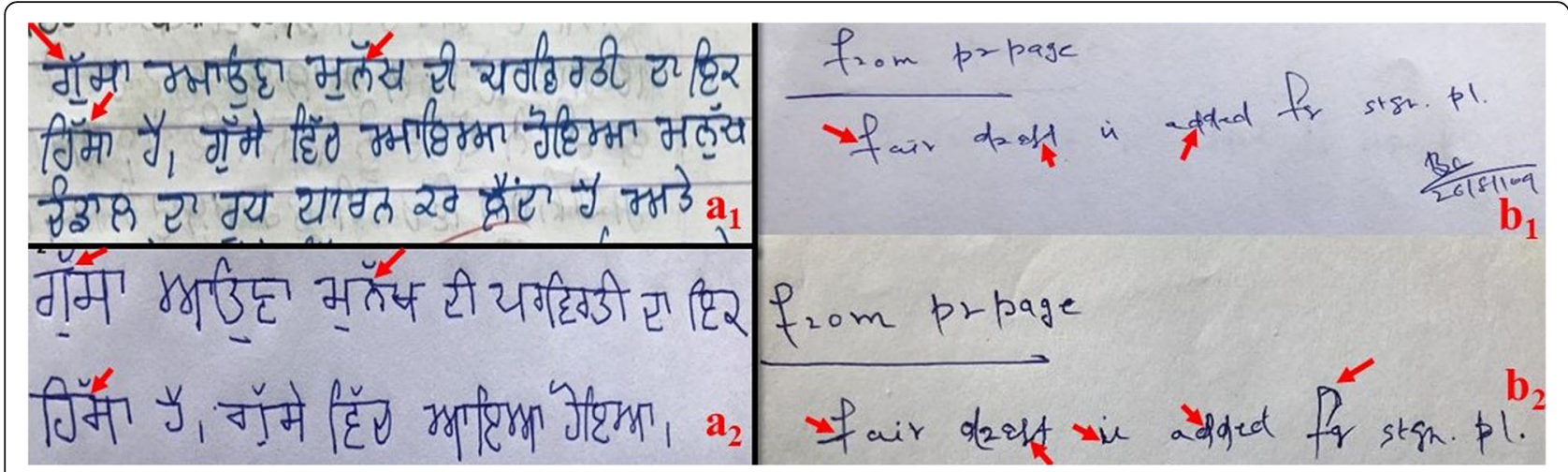

Fig. 3 Pen lifts and retouching/overwriting. $\mathbf{a}_{1}$ Handwriting before onset of RA showing consistency of strokes and no retouching/overwriting. $\mathbf{a}_{2}$ Inconsistent pen lifts in handwriting of RA patient. $\mathbf{b}_{\mathbf{1}}$ Absence of retouching to make correction in handwriting of RA patient. $\mathbf{b}_{\mathbf{2}}$ Extra lines to rejoin strokes at place of unnatural pen lifts along retouching and overwriting to make cautious corrections in copied writing

strokes with extra efforts in a concealing manner, indicating a conscious effort by the copier (Fig. 3b).

\section{Retouching and overwriting}

Retouching was not observed in any of the AH samples (Fig. 3a) and overwriting was found in only 16 of the 60 affected writings. Overwritten strokes in these writings were observed in bold form and some of these AH samples had instances of overwriting where letters had not been made properly by respective participants in the first attempt. Overall, RA did not show any significant effect on retouching and overwriting.

Whereas in the case of copied writings, retouching was observed at certain strokes as compared to the genuine AH samples (Fig. 3b). Bold overwriting was absent in these $\mathrm{CH}$ samples while drawing of strokes was observed at places of overwritten strokes in the AH samples. This signifies that the copying process was undertaken with cautious intentions (Fig. 5b).

\section{Letter formation}

Overall deterioration in letter formation was observed in 53 out of $60 \mathrm{AH}$ writings. The affected writings showed the presence of irregularities like an abrupt change in the curvature and hesitations, indicative of the poor quality of handwriting as opposed to their corresponding OH samples (Fig. 4a). Also, the overall formation of letters in the $\mathrm{AH}$ writings was more angular as compared to their corresponding $\mathrm{OH}$ samples; however, the execution of letters was similar in the movement of writings in both $\mathrm{OH}$ and $\mathrm{AH}$ samples.

For the copied writings, only a few samples showed a complete deterioration of strokes as compared to their corresponding genuine affected writings. Some of the $\mathrm{CH}$ samples contained drawing of exactly similar letter designs, punctuation marks, tremor, pen lifts, and certain other characteristics corresponding to their relative positions in the text, which is suggestive of the "rubber stamp effect". Nevertheless, the majority of copied writings showed an effort in making similar letter designs, but the difference in basic formation of letters indicated a different writing hand (Fig. 4b).

\section{Overall writing speed}

A significant decline in the speed of writing was observed in most of the handwriting samples $(n=41 / 60)$ written under the effect of rheumatoid arthritis as compared to the corresponding $\mathrm{OH}$ writings (Fig. 4a).

Strikingly, slow speed of writing was also observed in copied writings, indicated by the presence of hesitations or tremulous strokes at unexpected positions (Fig. 4b).

\section{Omission of letters/parts and words}

Omission of certain letters or parts of a few letters was observed in $28 \mathrm{AH}$ samples, suggestive of difficulty in the formation of some particular letters (Fig. 5a). This characteristic was absent in all the $\mathrm{OH}$ writing samples.

Copied writings for only a few subjects had omission of whole words along with a combination of various line quality defects (Fig. 5b). The copier even tried to copy the exact letter designs of genuine writings in some cases and skipped strokes at few positions while copying because of unfamiliarity with the exact formation of letters.

\section{Simplification/abbreviation of letters or parts of letters}

An increase in the simplification/abbreviation of writing was done by 24 out of the 60 RA patients; simplification of letters and even numerals was observed in these $\mathrm{AH}$ samples (Fig. 6a). This change may be attributed to the disruption of proper hand movement in forming particular letters because of the disease. However, the affected writings of a few participants also showed a proper and whole formation of letters and even words, despite the 


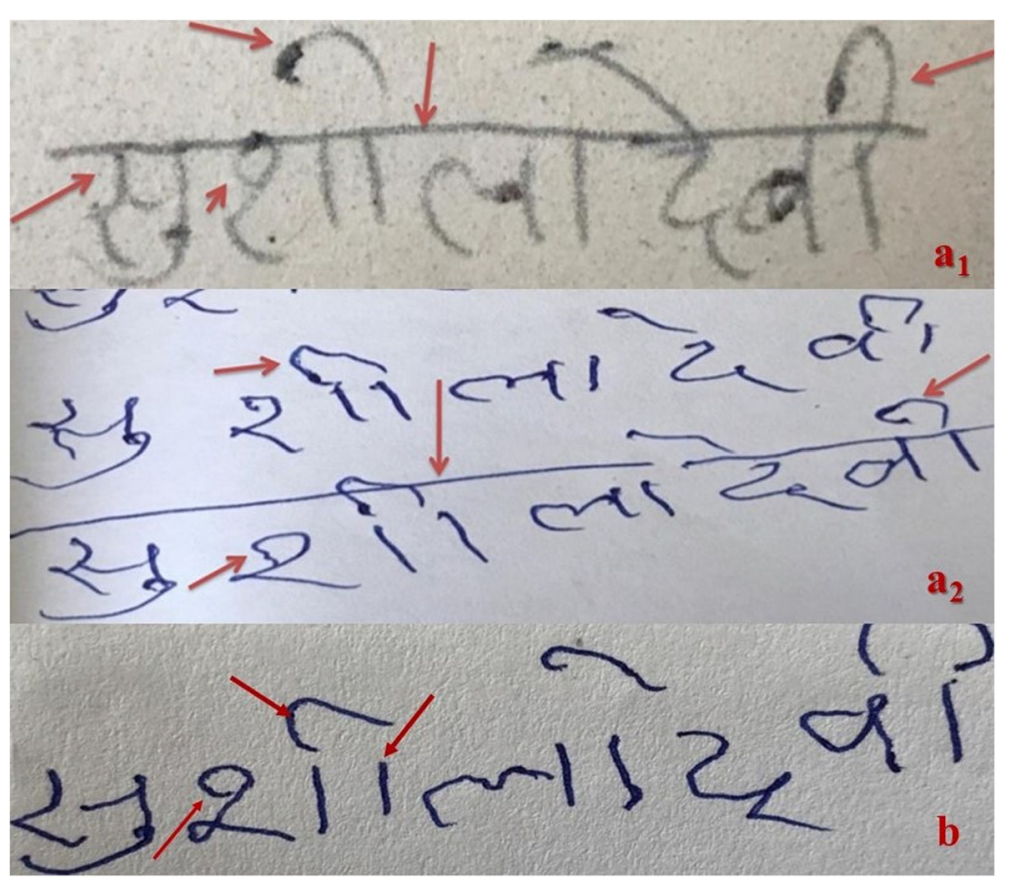

Fig. 4 Letter formation and writing speed. $\mathbf{a}_{\mathbf{1}}$ Handwriting before onset of RA showing no deterioration of letters and fast speed of writing. $\mathbf{a}_{\mathbf{2}}$ Deterioration of letter formation and slow speed in handwriting of RA patient. $\mathbf{b}$ Copied writing showing efforts in drawing deteriorated letters of affected writing along slow speed

execution of simplified/abbreviated words in their old writings. This indicates that these participants formed letters and words according to the copybook form even after the onset of illness, indicating their conscious efforts in forming whole and legible letters (Saini 2015).
On the contrary, simplification and abbreviation of writing were observed in only a few of the $\mathrm{CH}$ samples. The copier attempted to draw the simplifications observed in affected writings but was unable to simplify the letters or words properly in many copied writings

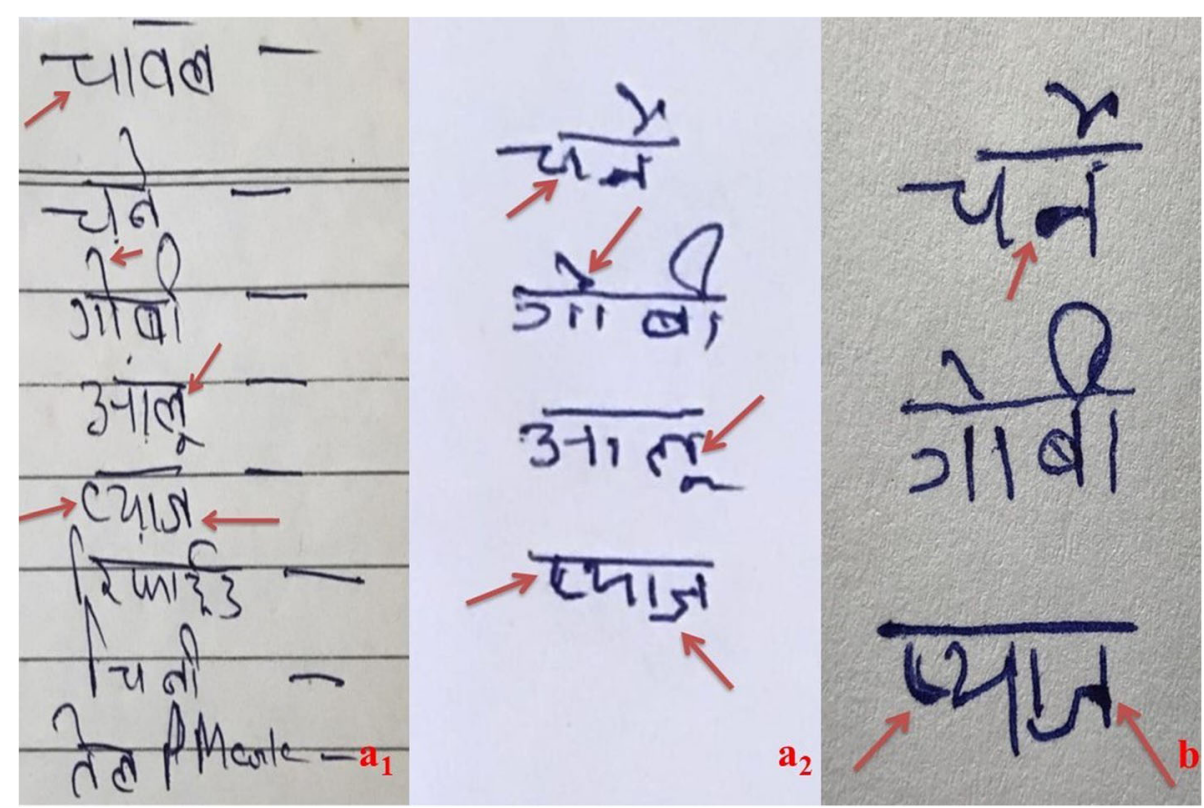

Fig. 5 Omission of letters and words. $\mathbf{a}_{\mathbf{1}}$ Handwriting executed before onset of RA. $\mathbf{a}_{\mathbf{2}}$ Omission of whole words and bold overwritten strokes in handwriting of RA patient. $\mathbf{b}$ Copied writing showing omission of a word and artificial drawing of overwritten strokes 


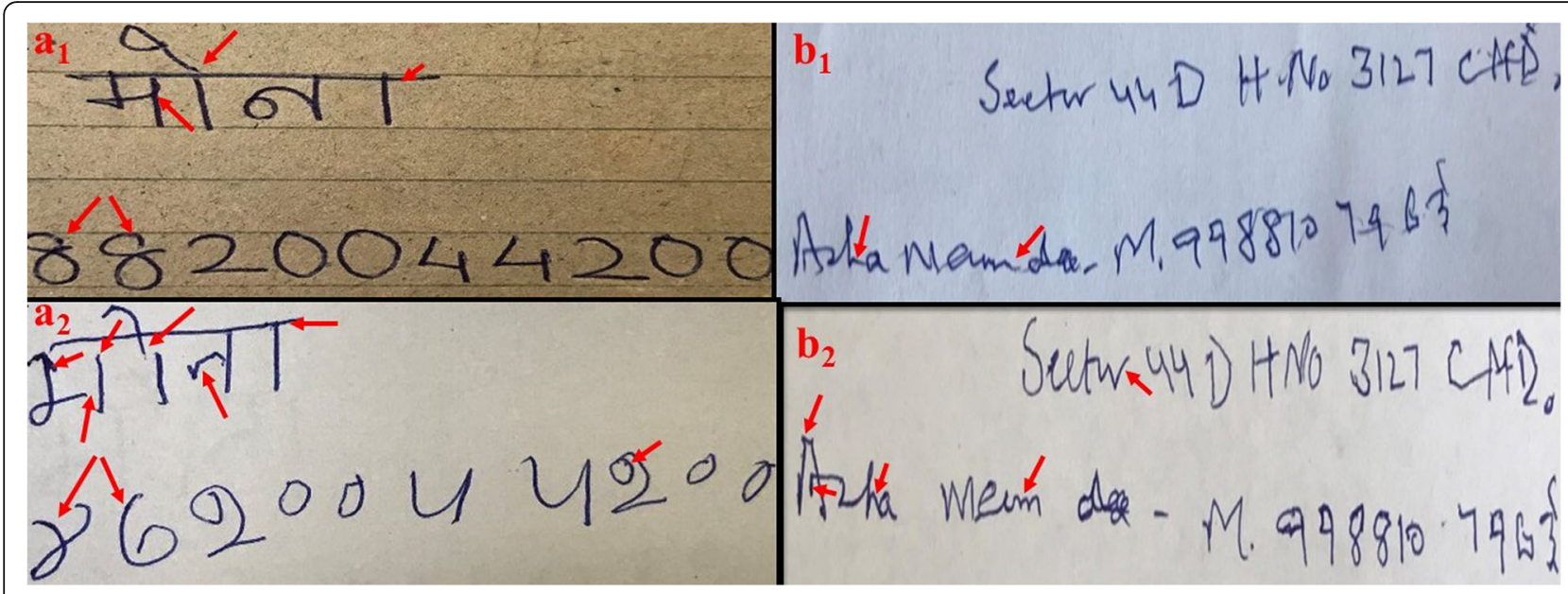

Fig. 6 Initial, terminal, and connecting strokes, simplification of words. $\mathbf{a}_{\mathbf{1}}$ Handwriting before onset of RA showing good quality of initial, terminal, and connecting strokes and no simplification. $\mathbf{a}_{\mathbf{2}}$ Simplified writing strokes, blunt initial and terminal strokes, and tremulous connecting strokes in handwriting of RA patient. $\mathbf{b}_{\mathbf{1}}$ Simplification of strokes and poor quality of initial, terminal, and connecting strokes in affected writing. $\mathbf{b}_{\mathbf{2}}$ Copied writing showing inability to simplify letters, hesitating initial and terminal strokes, and tremulous connecting strokes

(Fig. 6b). Thus, fully formed letter strokes were observed in most of the copied writings as compared to their corresponding genuine writings.

\section{Nature of initial and terminal strokes}

The presence of blunt $(n=18)$ and mixed type $(n=27)$ of initial and terminal strokes was observed in $45 \mathrm{AH}$ samples (Fig. 6a).

Blunt and hesitating type of initial and terminal strokes were also observed in copied writings, along with abrupt pen pauses at unexpected positions of letters, indicating the conscious efforts of the copier in starting and ending the letters (Fig. 6b).

\section{Nature of connecting strokes}

Tremulous types of connecting strokes were observed in 34 out of 60 affected writings. The connecting headline was missing in a few $\mathrm{AH}$ samples written in the Devanagari script. Few samples showed the presence of tremulous headlines above the words without joining them with the top of letters as compared to their corresponding old writings (Fig. 6a).

Tremulous types of connecting strokes were also in $\mathrm{CH}$ samples; however, they were at wrong and unexpected places (Fig. 6b). Also, most of the samples showed the presence of smooth strokes at unexpected places when compared to the affected writings.

\section{Overall size}

An increase in the overall size of writing (large size) was observed in the majority of AH writing samples $(n=47)$ as compared to their corresponding $\mathrm{OH}$ writings (Fig. 7a).
When compared to the corresponding $\mathrm{AH}$ samples, the maximum number of copied handwriting samples had medium-sized overall writing and was large-sized in only 1 sample (Fig. 7b).

\section{Slant, spacing, and alignment}

As compared to their $\mathrm{OH}$ samples, constancy in the slant (same as old writings) was observed in $51 \mathrm{AH}$ samples, narrow spacing between the words in 39 affected writings, and one half of the total affected writings showed variation in the alignment of overall writing (Fig. 8a).

On the contrary, only a few copied writings demonstrated a change in text alignment, along with the absence of other line quality defects (Fig. 8b). Otherwise, more than half of the copied writings were similar in the writing alignment, slant, and spacing for their corresponding affected writings.

The overall results of the qualitative analysis for $\mathrm{OH}$ vs AH reveal no significant effects of RA on handwriting characteristics such as retouching/overwriting and slant. Moreover, the results of qualitative analysis of $\mathrm{CH}$ vs $\mathrm{AH}$ indicate the minimal significance of handwriting characteristics like slant, spacing, and alignment while differentiating copied and genuine (affected) writings.

\section{Statistical analysis}

The results of the paired $t$-test showed an overall significant effect of RA $(p<0.05)$ on various handwriting characteristics (Table 3 ) including rhythm, tremor, pen lifts, letter formation, simplification, nature of initial and terminal strokes, nature of connecting strokes, the overall size of writing, writing speed, spacing, alignment, and omission of letters/words. On the other hand, handwriting characteristics of slant, retouching and overwriting 


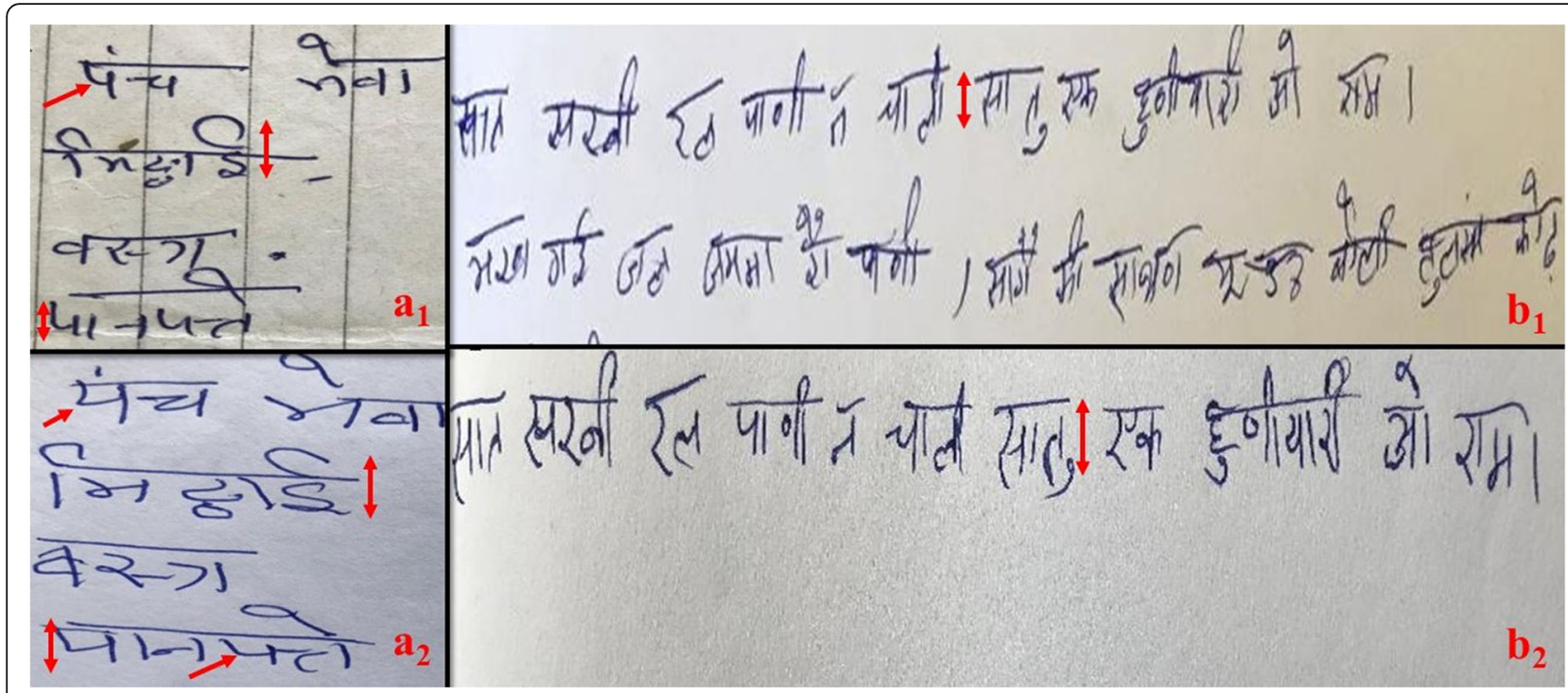

Fig. 7 Overall size. $\mathbf{a}_{\mathbf{1}}$ Handwriting before onset of RA showing medium writing size. $\mathbf{a}_{\mathbf{2}}$ Increased size in handwriting of RA patient. $\mathbf{b}_{\mathbf{1}}$ Mediumsized letters in handwriting of RA patient. $\mathbf{b}_{\mathbf{2}}$ Large size of letters in copied writing

did not show a statistically significant difference between the two groups $(p>0.05)$.

\section{Discussion}

Rheumatoid arthritis causes swelling, pain, and stiffness in hands, fingers, or wrist, thereby affecting the handwriting of an individual (Huber and Headrick 1999; Haberfehlner et al. 2011). The formation of angular strokes is suggestive of unbalanced movement impulses caused due to illness (Osborn 1929). However, the abrupt changes in the direction of strokes along with the momentarily slow speed of writing observed in the copied writings can be attributed to the bad line quality of writing (Osborn 1929; Harrison 1966). Moreover, a lack of control over the writing instrument was observed in copied writings as opposed to genuine writings.

Tremor was observed at curved strokes, straight strokes (mostly vertical), and in the loop formations (mostly "Matras" and numerals). This may be due to the lack of muscular control during the writing process caused by the illness (Ellen 1989; Saini 2015; Saini and Kaur 2019). Tremor of copied writings has been differentiated from the natural tremor (Osborn 1929; Brewester 1932; Harrison 1966; Hilton 1977, 1982; Saini 2015), as shown by the efforts made by the copier in drawing the design of tremor present in the affected writings. Thus, copied writings

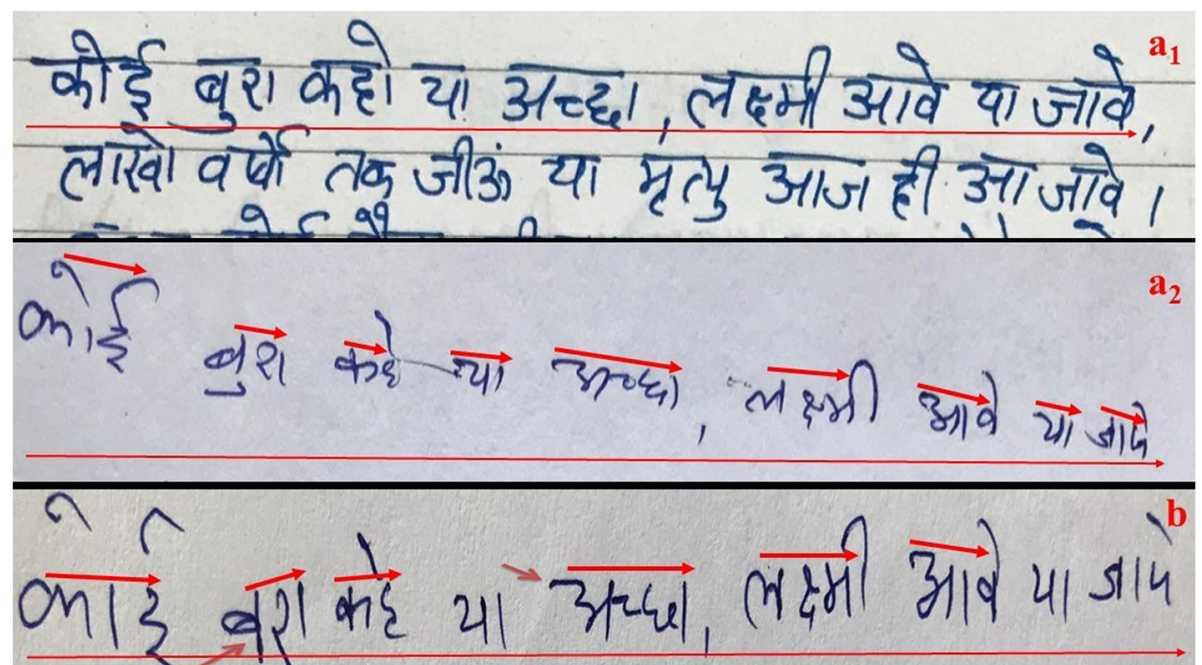

Fig. 8 Alignment. $\mathbf{a}_{\mathbf{1}}$ Handwriting before onset of RA showing mixed alignment. $\mathbf{a}_{\mathbf{2}}$ Change in overall alignment along downhill direction in handwriting of RA patient. $\mathbf{b}$ Mixed alignment in copied writing 
Table 3 Paired samples t-test results of characteristics in old vs RA affected handwriting samples

\begin{tabular}{|c|c|c|c|c|c|c|}
\hline Pair & Variables & Mean & Std. deviation & $t$ (test statistic) & $d f$ (degree of freedom) & Sig. (two-tailed) ( $p$-value) \\
\hline Pair 1 & RM_OH/RM_AH & -.483 & .504 & -7.429 & 59 & .000 \\
\hline Pair 2 & TM_OH/TM_AH & .183 & .390 & 3.639 & 59 & .001 \\
\hline Pair 3 & PN_OH/PN_AH & -.667 & .475 & -10.863 & 59 & .000 \\
\hline Pair 4 & RO_OH/RO_AH & -.100 & .303 & -2.560 & 59 & .013 \\
\hline Pair 5 & DL_OH/DL_AH & -.483 & .504 & -7.429 & 59 & .000 \\
\hline Pair 6 & WS_OH/WS_AH & -.217 & .415 & -4.040 & 59 & .000 \\
\hline Pair 7 & OM_OH/OM_AH & -.250 & .474 & -4.086 & 59 & .000 \\
\hline Pair 8 & SM_OH/SM_AH & -.517 & .504 & -7.942 & 59 & .000 \\
\hline Pair 9 & IT_OH/IT_AH & -.683 & .469 & -11.283 & 59 & .000 \\
\hline Pair 10 & NC_OH/NC_AH & -.817 & .390 & -16.212 & 59 & .000 \\
\hline Pair 11 & SI_OH/SI_AH & -.150 & .360 & -3.227 & 59 & .008 \\
\hline Pair 12 & SL_OH/SL_AH & -.933 & .252 & -28.740 & 59 & .000 \\
\hline Pair 13 & SP_OH/SP_AH & -.650 & .481 & -10.468 & 59 & .000 \\
\hline Pair 14 & AG_OH/AG_AH & -.467 & .503 & -7.185 & 59 & .000 \\
\hline
\end{tabular}

Results of paired $t$-test with mean values of differences in "OH" and "AH", standard deviation values, significance values (two-tailed), $t$-values, and degree of freedom obtained from statistical analysis of handwriting characteristics of RA subjects

indicated towards conscious act with strong muscular coordination as compared to the corresponding genuine writings with lack of muscular control.

Pen lifts were observed at only certain strokes, specifically at turning positions of the letters and in words with bigger letter formations which may be largely due to the muscular pain. Thus, the position of the pen lifts can indicate the actual health condition of the writer (Osborn 1929; Harrison 1966; Hilton 1977, 1982; Saini 2015). On the other hand, pen lifts in copied writings have been observed in the form of hesitation marks in combination with the other line quality defects (Harrison 1966; Saini 2015).

Overwriting in affected writings has often been observed to be performed in a fearless, clear, and bold manner despite being written under the effect of illness, thus, signifying the genuineness of writing (Osborn 1929; Brewester, 1932 1950; Harrison 1966; Saini 2015). Whereas, in the case of copied writings, retouching was observed at certain strokes in a concealed manner to make the strokes look like those of genuine writings. Moreover, bold overwritten strokes were found to be absent in copied writings.

Deterioration in the formation of letters in varying degrees has been observed in the majority of affected writings (Koppenhaver 2007; Sharma 2012). The letter strokes have not been found to be smooth due to the muscular rigidity caused by arthritis (Sulner 1966). Also, it may have caused inhibition of proper hand movement required in forming particular letters. In the case of copied writings, few samples showed deterioration of strokes, while a few other samples showed conscious efforts in forming identical letter designs.
Furthermore, the speed of handwriting has frequently been observed to decrease in almost all affected handwriting samples (Hilton 1977; Saini 2015). The affected handwriting samples have been observed to show illegible, scratchy, and angular strokes, which is possibly due to the difficulty in hand movements (Osborn 1929, Kumar 1989). In our study, the omission of letters or their parts was observed in almost half of the AH samples. This feature may have been due to the stiffness of muscles involved in the writing process, leading to difficulty in making certain letters. Omission of letters was also observed in a few $\mathrm{CH}$ samples along with the combination of various line quality defects.

The formation of letters demonstrated more angularity and slow speed, along with simplification and abbreviation of letters/words in the affected writing samples. This may be attributed to the swelling and joint deformities caused by RA. Simplifications observed in the $\mathrm{CH}$ samples may be due to the unfamiliarity about the writing movements instead of a lack of muscular inhibition.

Initial and terminal strokes were found to be blunt and executed with difficulty in only a few samples, whereas the remaining samples showed the execution of a mixture of both tapered and blunt strokes. Also, tremulous types of connecting strokes were observed in a relatively angular and zig-zag form in the majority of affected writings.

Previous studies have reported variations in the overall size of letters in the majority of affected writings (Osborn 1929; Hilton 1956, 1977; Miller, 1987; Saini 2015; Saini and Kaur 2019). Our results showed that an increase in the size of writing was observed in most of the affected writings. Also, an extreme change in the size of copied writings was observed as compared to the 
corresponding affected writings, making the visual appearance of the letters in the copied writings to be remarkably different from their AH counterparts.

The slant of writing was the most unaffected characteristic in the case of AH samples. Slant is said to be one of the fixed writing habits and even a slight divergence in a few strokes may cause the lack of genuineness (Osborn 1929). Furthermore, a change in the alignment of handwriting was also observed, which may be due to a difference in the movement and position of the writing arm with respect to the line of writing (Osborn 1929; Harrison 1966; Saini 2015). A decrease in the spacing was observed in the majority of affected writings, which may be attributed to the difficulty in moving the writing instrument.

Lastly, the statistical analysis to compare both the genuine writings, $\mathrm{OH}$ versus $\mathrm{AH}$, has demonstrated statistical significance for the majority of handwriting characteristics. This indicates that a well-marked difference exists among the writings written under two different conditions, that is, before and after the onset of illness (Saini and Kaur 2019).

\section{Conclusion}

It can be concluded that rheumatoid arthritis brings about considerable effects on the handwriting of an individual owing to the development of deformities and severe pain in the hand joints. However, the writing of a person affected by RA can be distinguished from copied writings by identifying non-genuine features, such as artificial tremor, hesitations, delicate retouching, unnatural pen lifts, and the addition of peculiar writing characteristics of the copier. Meticulous assessment of these peculiarities may help document examiners to determine the genuineness of any questioned document. The findings of the study will be helpful for document examiners in solving the relevant cases; we also suggest that document examiners be well-acquainted with the knowledge of factors that may affect handwriting characteristics.

\section{Abbreviations}

RA: Rheumatoid arthritis; $\mathrm{OH}$ : Handwriting samples collected from subjects before the onset of rheumatoid arthritis; $\mathrm{AH}$ : Handwriting samples collected from subjects after the diagnosis of rheumatoid arthritis; $\mathrm{CH}$ : Copied writings prepared with the help of volunteer

\section{Acknowledgements}

Not applicable

\section{Authors' contributions}

The research study was designed by KS. BS performed collection and analysis of handwriting samples. MK contributed in the writing of manuscript. All the authors contributed in the interpretation, compilation, and presentation of the results. The authors read and approved the final manuscript.

\section{Funding}

Not applicable
Availability of data and materials

All the data generated or analyzed during this study are included within this article and its supplementary files.

\section{Declarations}

\section{Ethics approval and consent to participate}

Before collection of handwriting samples, the subjects were informed about the nature of the study and written consent was obtained from all the subjects, stating "Collected handwriting samples will be solely used for the purpose of research and shall not be misused". Ethical approval to collect handwriting samples was granted by Institutional Ethical Committee (IEC), Punjabi University (Patiala); vide letter no. 167/SEC, dated 17 February 2020.

\section{Consent for publication}

Not applicable

\section{Competing interests}

The authors declare that they have no competing interests.

Received: 20 August 2020 Accepted: 13 August 2021

Published online: 24 August 2021

References

Baker JN (1950) Law of disputed and forged documents. The Michie Company, Charlottesville

Brewester F (1932) Contested documents and forgeries. The Book Company Ltd., Calcutta

Ellen D (1989) The scientific examination of documents: methods and techniques. Ellis Horwood Ltd., Chichester

Haberfehlner H, Visser B, Daffertshofer A, Rossum MA, Roorda LD, Dekker J, Hoeksma A (2011) Handwriting difficulties in juvenile idiopathic arthritis: a pilot study. Clinical and experimental rheumatology 29(5):887-893

Harrison WR (1966) Suspect documents: their scientific examination. Sweet and Maxwell Ltd., U.K

Hilton O (1956) Influence of serious illness on handwriting identification. Postgrad Med, 19(2), 36-48

Hilton O (1977) Influence of age and illness on handwriting: identification problems. Forensic Science 9(3):161-172. https://doi.org/10.1016/0300-9432 (77) $90087-5$

Hilton O (1982) Scientific Examination of Questioned Documents (Revised ed.). Elsevier Science Publishing Co., New York.

Huber RA, Headrick AM (1999) Handwriting identification: facts and fundamentals. CRC Press LLC, Florida, Boca Raton. https://doi.org/10.1201/ 9781420048773

Koppenhaver KM (2007) Forensic document examination. Humana Press. 978-159745-301-1. https://doi.org/10.1007/978-1-59745-301-1

Kumar K (1989) Identification of disputed documents, fingerprints and ballistics, 4th edn. Eastern Book Co, Delhi

Miller LS (1987) Forensic examination of arthritic impaired writing. Journal of Police Science and Administration 15(1):51-55

Osborn AS (1929) Questioned documents, 2nd edn. Boyd Publishing Co, Albany, NY

Quirke AJ (1930) Forged, anonymous and suspect documents. George Routledge and Sons Ltd., London

Saini K (2015) A study of various factors influencing handwriting. Lambert Academic Publishing, Patiala

Saini K \& Kaur M (2019) Forensic study on the effect of age and illness (Parkinsonism) on handwriting characteristics. Egypt J Forensic Sci, 9(1), 24. https://doi.org/10.1186/s41935-019-0131-9

Sharma BR (2012) Handwriting Forensics. Universal Law Publishing - An imprint of Lexis Nexis. 10: 9350358425

Sulner HF (1966) Disputed documents. Oceana Publications Inc, New York

\section{Publisher's Note}

Springer Nature remains neutral with regard to jurisdictional claims in published maps and institutional affiliations. 